\title{
Adaptation Measurement of CAD/CAM Dental Crowns with X-Ray Micro-CT: Metrological Chain Standardization and 3D Gap Size Distribution
}

\author{
L. Tapie, ${ }^{1,2}$ N. Chiche, ${ }^{2}$ P. Boitelle, ${ }^{2}$ P. Morenton, ${ }^{3}$ J.-P. Attal, ${ }^{2}$ N. Schmitt, ${ }^{4,5}$ and E. Vennat ${ }^{6}$ \\ ${ }^{1}$ IUT de Saint Denis, Paris 13 University, Sorbonne Paris Cité University, Place du 8 mai 1945, 93206 Saint Denis Cedex, France \\ ${ }^{2}$ Department of Biomaterials, URB2i, EA 4462, Faculty of Dental Surgery, Paris-Descartes University, Sorbonne Paris Cité University, \\ 1 rue Maurice Arnoux, 92120 Montrouge, France \\ ${ }^{3}$ Laboratoire du Génie Industriel, EA 2606, Centrale-Supélec, Grande Voie des Vignes, 92295 Chatenay-Malabry Cedex, France \\ ${ }^{4}$ LMT-Cachan, ENS Cachan, CNRS UMR 8535, PRES UniverSud, 61 avenue du Président Wilson 94235 Cachan Cedex, France \\ ${ }^{5}$ University Paris-Est Créteil Val-de-Marne, Place du 8 mai 1945, 93206 Saint Denis Cedex, France \\ ${ }^{6}$ Laboratoire Mécanique des Sols, Structures et Matériaux, CNRS UMR 8579, Ecole Centrale Paris, Grande Voie des Vignes, 92295 \\ Chatenay-Malabry Cedex, France
}

Correspondence should be addressed to L. Tapie; laurent.tapie@univ-paris13.fr

Received 2 September 2016; Accepted 17 October 2016

Academic Editor: Gianluca Percoco

Copyright (C) 2016 L. Tapie et al. This is an open access article distributed under the Creative Commons Attribution License, which permits unrestricted use, distribution, and reproduction in any medium, provided the original work is properly cited.

\begin{abstract}
Computer-Aided Design and Manufacturing systems are increasingly used to produce dental prostheses, but the parts produced suffer from a lack of evaluation, especially concerning the internal gap of the final assembly, that is, the space between the prepared tooth and the prosthesis. X-ray micro-Computed Tomography (micro-CT) is a noninvasive imaging technique enabling the internal inspection of the assembly. It has proved to be an efficient tool for measuring the gap. In this study, a critical review of the protocols using micro-CT to quantify the gap is proposed as an introduction to a new protocol aimed at minimizing errors and enabling comparison between CAD/CAM systems. To compare different systems, a standardized protocol is proposed including two reference geometries. Micro-CT is used to acquire the reference geometries. A new 3D method is then proposed and a new indicator is defined (Gap Size Distribution (GSD)). In addition, the usual 2D measurements are described and discussed. The 3D gap measurement method proposed can be used in clinical case geometries and has the considerable advantage of minimizing the data processing steps before performing the measurements.
\end{abstract}

\section{Introduction}

Computer-Aided Design and Manufacturing (CAD/CAM) systems are becoming common in dental prosthesis production [1-4]. The adaptation of prosthesis, on the remaining and prepared dental tissues, obtained by CAD/CAM is a key factor that must be considered carefully to ensure the longevity and survival of the tooth-restoration assembly [5]. Prosthesis adaptation focuses on the metrological measurement of the fit between the prepared tooth to be restored and the manufactured restoration $[6,7]$. A fit less than 100 to $120 \mu \mathrm{m}$ is clinically validated $[8,9]$ and helps in limiting the restored tooth morbidity, gingival irritation, secondary caries, and sealing cement dissolution $[10,11]$. This fit is considered as the most clinically relevant parameter to assess CAD/CAM system accuracy. Thus, assessing the accuracy of dental CAD/CAM systems is becoming an important topic in restorative dentistry.

In parallel, imaging techniques are more and more powerful and enable us to investigate the dental structures at different scales from the whole facial geometry of a patient $[12,13]$ to the dental tissues microstructure [14]. X-ray microComputed Tomography (micro-CT) is considered reliable for imaging dental structures [15] and more specifically for metrological measurements of dental prosthesis adaptation, and protocols to assess crown adaptation with micro-CT can 


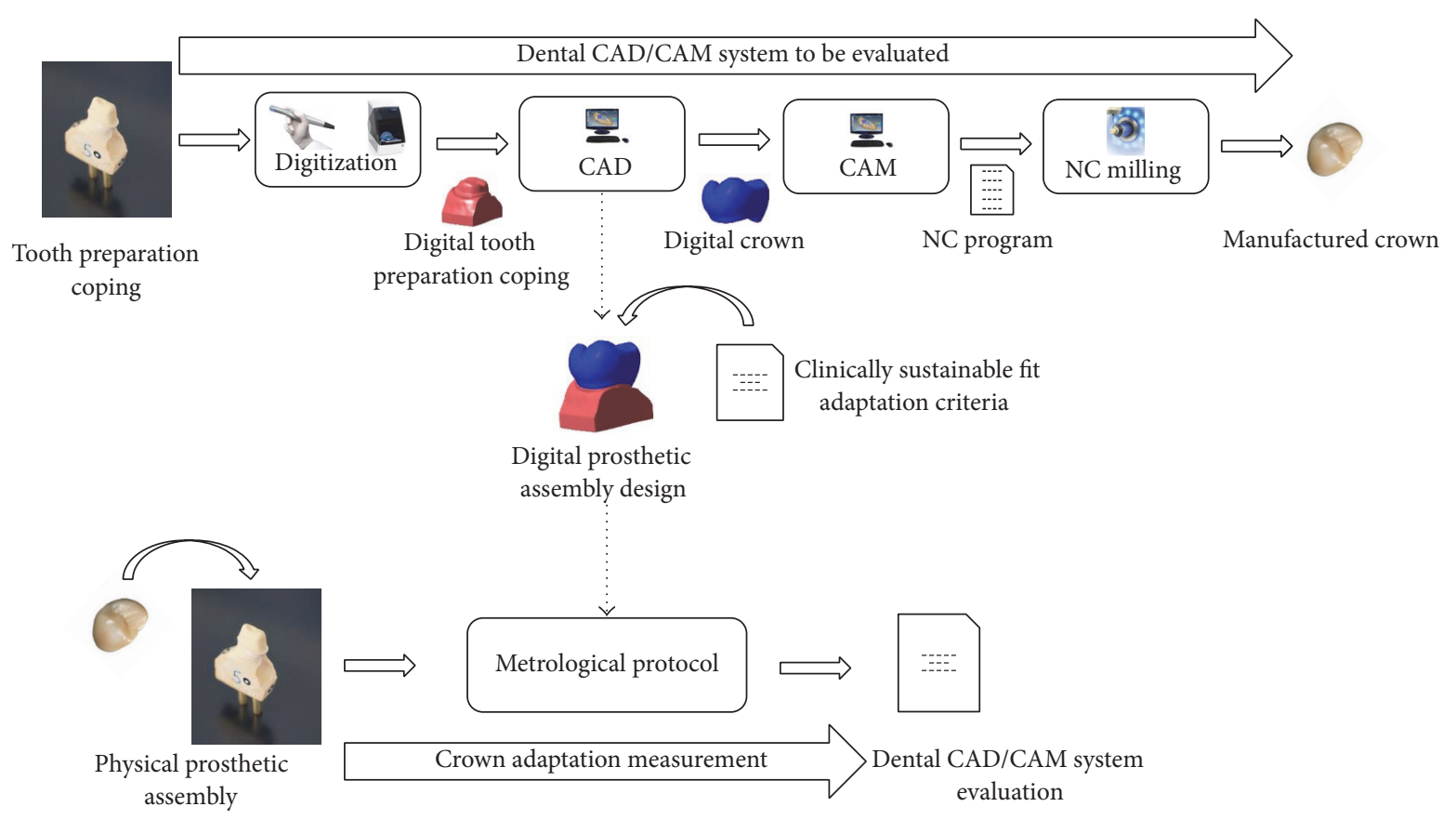

FIGURE 1: Dental CAD/CAM assessment workflow.

be found in the literature [16-19]. Due to the possibility of having access to the inner geometry of the dental system, micro-CT imaging is a relevant measurement technique for acquiring the fit of the assembly between the crown and the prepared tooth (die). The gap space to be measured is overlapped by the crown and cannot be reached by a contact probe or classical noncontact optical measurement devices without manipulating the assembly elements [1921]. Thus, the micro-CT technique eliminates in particular the measurement uncertainties introduced by handling the assembly. Nevertheless, this protocol must be optimized and standardized as certain measurement biases are introduced involving fit values ranging from 20 to $200 \mu \mathrm{m}$. This means that the results cannot be compared and may not represent the true values for adaptation.

The aim of this article is to validate and optimize a metrological measurement protocol of CAD/CAM dental crown fits with micro-CT and to standardize such measurements. First, we propose a detailed analysis of the metrological chain involved by micro-CT scanning applied to the crown adaptation assessment. Then, we present a pilot experimental study to standardize the crown adaptation measurements and, finally, we discuss the results of our experiments. A new indicator called Gap Size Distribution (GSD) is defined to characterize gap geometry. It can be used for standardized geometries and clinical cases.

\section{On Dental CAD/CAM Assessment}

2.1. Objectives. The assessment of dental CAD/CAM systems is carried out by the metrological measurement of the fit between a coping model of a prepared tooth (die) and the restoration (crown prosthesis) manufactured using CAD/CAM (Figure 1).

As illustrated in Figure 1, the dental CAD/CAM process is divided into three main steps: (1) a coping of a tooth preparation is digitized; (2) a restorative prosthetic assembly is designed on a computer (computer aided design (CAD)) according to the clinical recommendation for fit adaptation; (3) the prosthesis milling process is performed with Computer-Aided Manufacturing (CAM) and numericalcontrolled (NC) milling.

After the CAD/CAM process, the prosthesis coping and tooth preparation coping assembly is measured to assess prosthesis adaptation. A metrological protocol is needed to perform the fit adaptation measurement. The objective is to evaluate the gap between the prepared tooth coping and the prosthesis coping on the basis of dimensional criteria described in Section 3.2.

As the best fit adaptation measurement is evaluated through the dimensions of the gap between the tooth preparation coping and the prosthesis coping, it is necessary to access the gap volume. As the prosthesis shape covers this volume, micro-CT imaging seems to be a relevant data acquisition technique to access the gap dimensions [22].

2.2. Crown Adaptation Criteria. The terminology of crown adaptation criteria that has been unanimously accepted was defined by Holmes et al. [23] and is illustrated in Figure 2.

The measurements of the distances are usually made in planes (Figure 2(a)) containing the prosthesis axis of insertion (prosthesis path of draw in Figure 2). According to the glossary of prosthodontic terms [24], the prosthesis path 


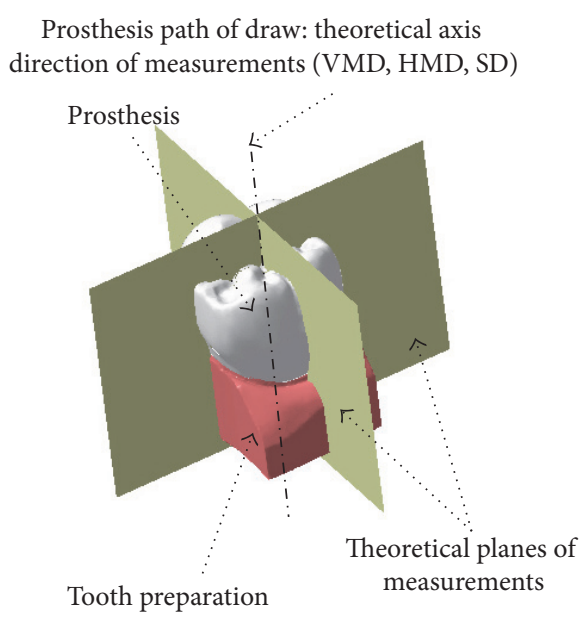

(a)

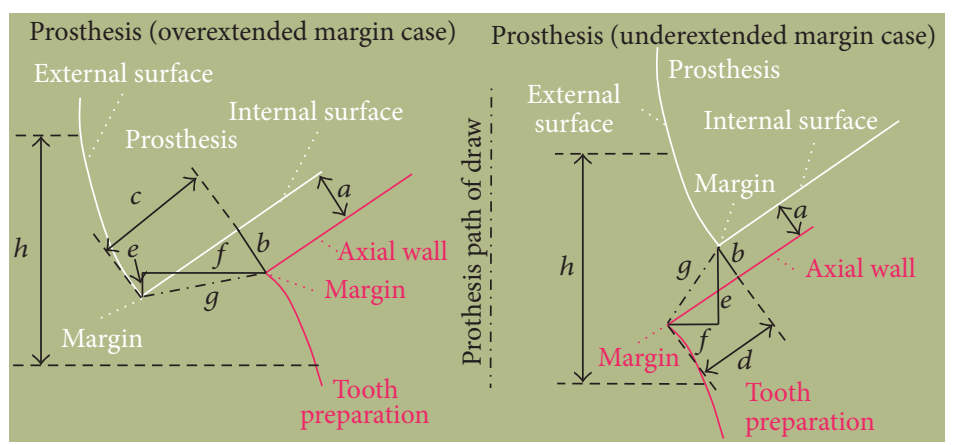

(a) Internal gap (IG)

(b) Marginal gap (MG)

(c) Overextended margin $(\mathrm{OM})$

(d) Underextended margin (UM) (e) Vertical margin discrepancy (VMD)

( $f$ ) Horizontal margin discrepancy (HMD)

(g) Absolute margin discrepancy (AMD)

(h) Seating discrepancy

(b)

FIgURE 2: Definitions of adaptation criteria from Holmes et al. [23].

of draw is a synonym of path of placement or path of insertion or path of withdrawal and can be defined as the specific direction in which a prosthesis is placed on the abutment teeth or dental implant(s). According to this definition the prosthesis axis (PA) is defined as the planned line or path of placement and removal for a dental restoration.

In clinical practice, either in conventional (manual) or in $\mathrm{CAD} / \mathrm{CAM}$ prosthesis production, the $\mathrm{PA}$ is materialized by a fictive axis defined in the dentist mind during manual milling of the tooth. Then, the PA is recognized by anatomical surface analysis of the milled prepared tooth during prosthesis design tasks. In the case of CAD/CAM production, a manual identification of PA may be assisted by computerized detection of a geometrical axis minimizing the undercuts area. Nevertheless, the PA definition is too vague to clearly/mathematically identify a unique PA resulting in several possible PA identifications for the same tooth preparation.

As the crown covers the tooth preparation, the internal gap is defined to evaluate the distance between the prepared tooth and the crown.

(a) Internal gap $(I G)$ is perpendicular distance from the internal surface of the prosthesis to the axial wall of the tooth preparation.

Depending on the manufacturing and the clinical adjustment, the crown margin may overextend or underextend the tooth preparation margin as shown in Figure 2(b) so several criteria are defined to evaluate overextension or underextension on the marginal area.

(b) Marginal gap $(M G)$ is perpendicular distance from the internal surface of the prosthesis to the margin of the tooth preparation.

(c) Overextended margin $(O M)$ is perpendicular distance from the marginal gap (MG) to the prosthesis margin. (d) Underextended margin (UM) is perpendicular distance from the marginal gap (MG) to the margin of the tooth preparation.

(e) Vertical marginal discrepancy (VMD) is distance parallel to the path of draw of the prosthesis from the margin of the prosthesis to the margin of the tooth preparation.

(f) Horizontal marginal discrepancy (HMD) is distance perpendicular to the path of draw of the prosthesis from the margin of the prosthesis to the margin of the tooth preparation.

(g) Absolute marginal discrepancy (AMD) is absolute distance from the prosthesis margin to the margin of the tooth preparation. When OM or UM are null, AMD is equal to MG. When MG is null, AMD is equal to OM or UM.

(h) Seating discrepancy (SD) is distance parallel to the path of draw through an arbitrary point (or points) on the external surface of the prosthesis and tooth preparation away from the margins.

Fit adaptation measurement protocols based on microCT scanning have been proposed in the literature [6].

\section{Critical Analysis of Existing Protocols Using Micro-CT}

In order to process the data acquisition it is necessary to define the geometrical systems of reference (plane and direction to perform the measurements) and the specificities of the micro-CT scan measurements. These technical aspects are dealt with to a greater or lesser degree in the description of existing protocols.

3.1. The Issue of Matching and Identifying Acquisition and Prosthesis Axis. The principle of micro-CT tomography consists in the mathematical reconstruction of a complete 


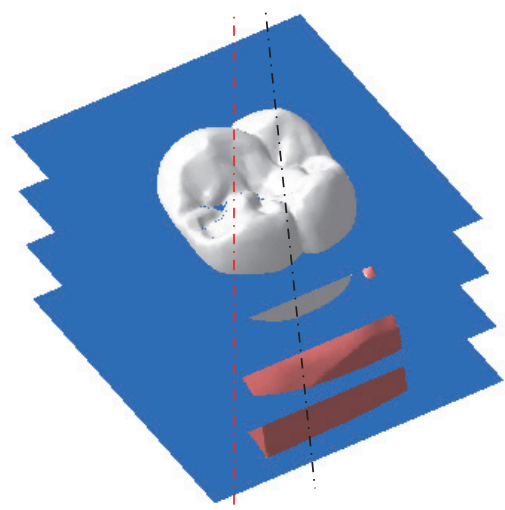

-..- CT scan axis of acquisition (AA: micro-CT scan rotation axis)

... - Theoretical axis of prosthesis insertion (PA)

(a)

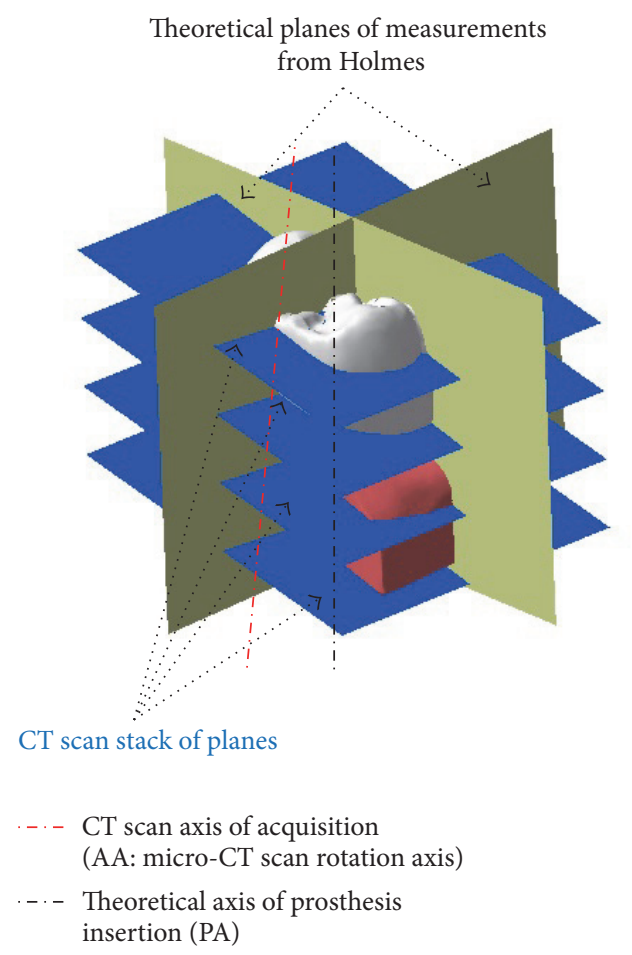

(b)

FIGURE 3: Directions of acquisition versus theoretical directions of measurements.

volume from a series of several hundred of projection images (radiograph or radiograms) obtained by illuminating with an X-ray source an object from different directions and collecting the transmitted data thanks to a detector [25]. Either the X-ray source or the detector (linear or array detector) is moving during the exposure around the stationary object (rotation around an axis) or they are stationary and the object is rotating around the axis. In some case an additional sliding movement is added to the rotation movement. The reconstructed images are obtained from the scan data by mathematical processing (solving the inverse Radon transformation using filtered back projection or linear algebra or iterative physical model-based maximum likelihood expectation maximization). Images are digitally displayed in terms of relative radiodensity and archived as a stack of images in parallel planes (blue planes in Figure 3(a)). These planes are perpendicular to the rotation axis of the micro-CT scan, which can be termed the axis of acquisition (AA).

The first issue raised by existing protocols is matching and identifying acquisition (AA) and prosthesis axis (PA). In the academic protocols proposed by Borba et al. [16], Pelekanos et al. [17], and Mously et al. [18], AA is considered as parallel to PA whereas they were not aligned during images acquisition. Then, the PA identification is performed on the stack of images acquired (Pelekanos et al. [17]) or on a 3D volumetric reconstruction of the data acquired (Borba et al. [16]), then generating a stack of slices around this axis (beige vertical planes in Figure 3(b)). Thus, the resulting slicing planes of measurements are processed around an axis inclined from the axis of insertion (PA), involving measurement errors in the adaptation criteria. Besides, a manual identification of $\mathrm{PA}$ is performed, so PA axis identification cannot be unique and cannot be the same PA defined during the crown design (black axis in Figure 3).

For each protocol the directions of slicing planes of measurements around PA cover the buccolingual (width direction of the tooth as illustrated in Figure 4) and mesiodistal directions (length direction of the tooth as illustrated in Figure 4). Nevertheless, in Pelekanos et al. [17] and Borba et al. [16] protocols, respectively, ten and two radial slicing planes are used for measurements and in Mously et al. protocol five parallel planes around PA are used. As buccolingual and mesiodistal directions are anatomical directions $[26,27]$, their identification remains difficult. For instance, IG parameter must be measured perpendicular to the tooth preparation surface whereas the slicing planes directions are not perpendicular to these surfaces as illustrated in Figure 4.

The measurement reference system obtained after data processing can be not coincident with the expected theoretical measurement reference system due to the unclear identification of PA and measurements directions after acquisition. As mentioned previously, it is necessary to standardize the definition of PA.

3.2. The Issue of Identifying and Measuring the Gap Geometry. The images quality and accuracy might be affected by acquisition artifacts and the spatial resolution of the micro-CT scan [28]. The beam-hardening effect introduces enhanced 


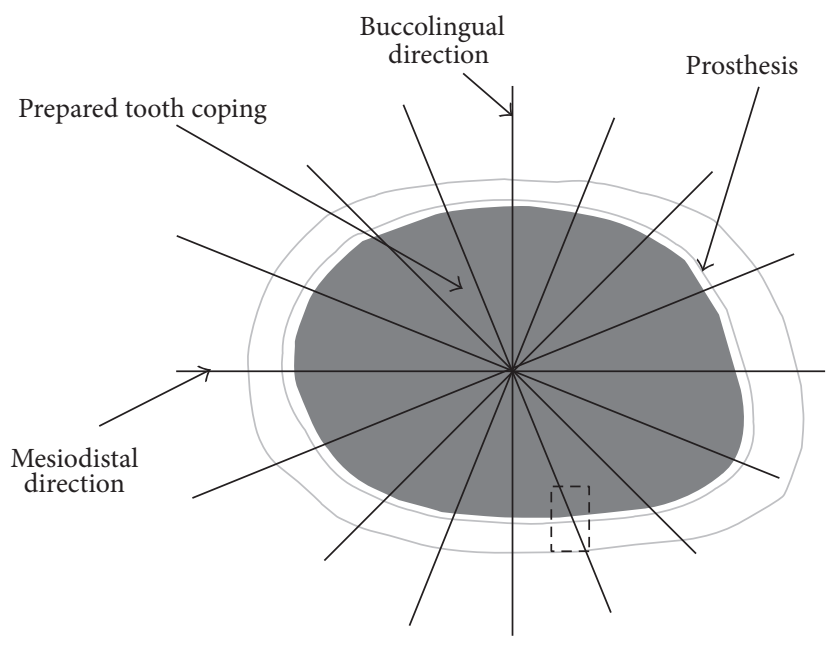

(a) Set of radial reslices directions

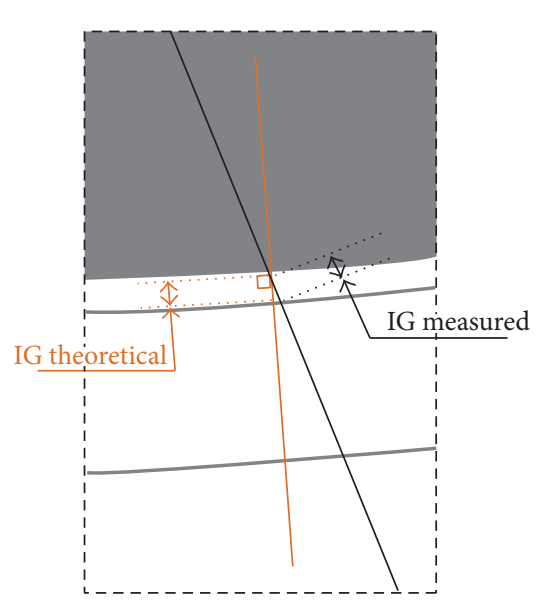

(b) Measurement error

FIGURE 4: Set of radial slicing planes containing PA (a) planes directions and (b) measurement error.

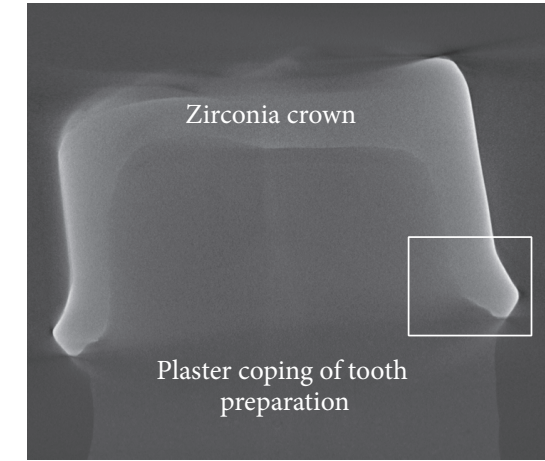

Contrast problem due to the difference in radioopacity between the crown and coping material

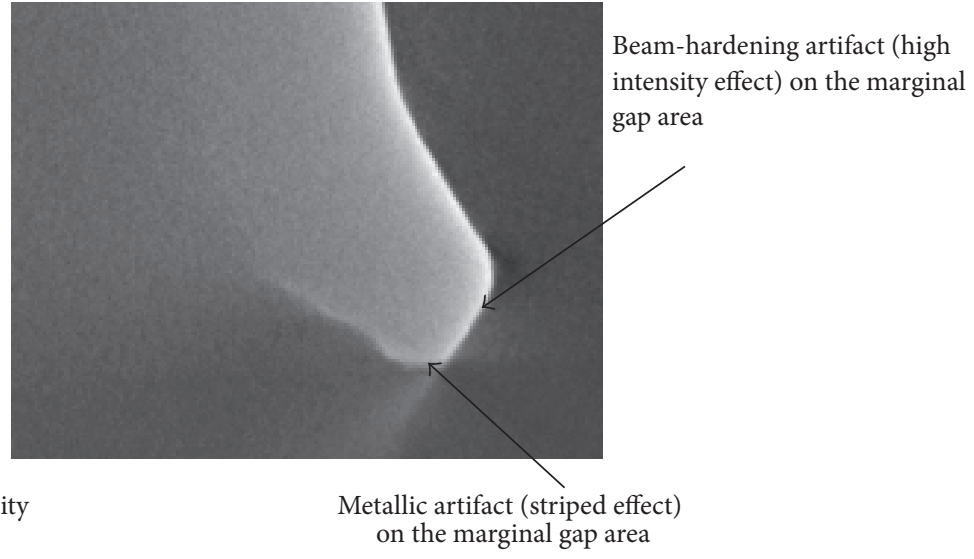

FIGURE 5: Acquisition artifacts. intensity on the object border [29]. Such artifacts are closely linked to the polychromatic property of the X-ray beam. A metallic part (aluminum, copper, or tungsten filter) can be used for filtering the beam and attenuating the beamhardening effect. However, such metallic filtering increases acquisition noise. The ring effect introduces concentric circles on images, centered on the micro-CT scan rotation axis. This effect can be imputed to mechanical displacement defects or detector defects (defective pixels) during X-ray radiographic projections. Such defect effects can be avoided by modifying the scanning strategy (minimization of the influence of defective pixels on radiographic projections) or specific image-processing algorithms applied on the reconstructed stack of images. The presence of metal on the object to be scanned can generate stripes on the reconstructed images. This effect is due the high density of the metallic object, which is higher than the density that the X-ray beam can penetrate. The object or X-ray source and detector movements during acquisition introduce shadows on the reconstructed images.
As it was presented in Section 2, to measure the gap on the prosthetic assembly, the prosthesis and prepared tooth copings boundaries must be visible, accurate, and dimensionally reliable: artifacts on reconstructed images must be avoided and the contrast must be sufficient to identify the prosthetic assembly boundaries. In the case of dental crown adaptation measurements, the radiodensity and composition of the materials used for both the crown and the coping of the prepared tooth must be considered. The range of biomaterials used for CAD/CAM crown manufacturing is very wide and includes ceramics, composites, hybrids, and polymers $[28,30]$. These different types of material vary due to their composition and radiodensity, leading to specific $\mathrm{X}$-ray beam adjustment and filtering. In Figure 5, an X-ray scan of a prosthetic assembly composed of a zirconia crown and a plaster coping is illustrated; nonsuitable adjustment and filtering involve acquisition artifacts and make image processing difficult: the radiodensity properties of these two materials are too different to allow clear distinguishing of the assembly gap. 


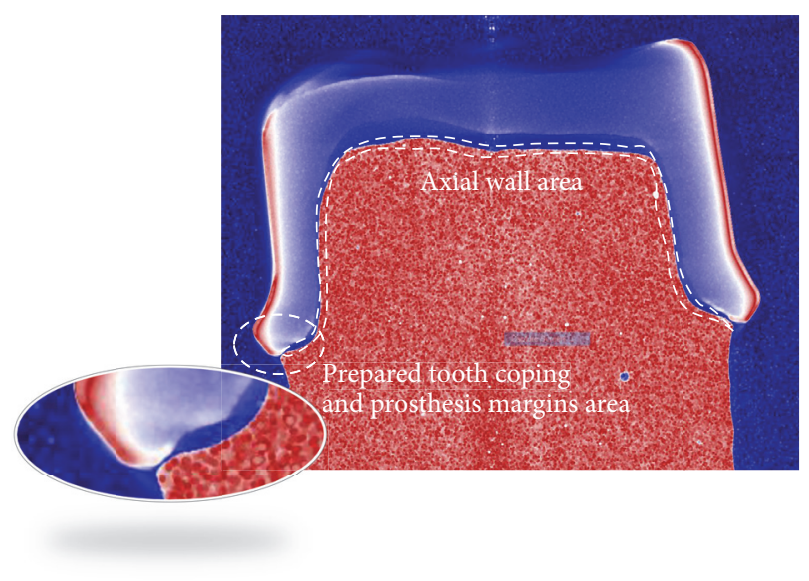

Figure 6: Anatomical element recognition.

Another problem involved by micro-CT scan protocol is the manual identification of the anatomical elements (tooth and preparation margins, tooth axial wall, and prosthesis internal surface) necessary to perform the measurement of the fit adaptation criteria. As illustrated by a colored X-ray image in Figure 6, the 2D curve limitation associated with the axial wall is very difficult to identify; the position of the points associated with the margins are not clear due to the fillet radius. As a consequence, manual recognitions of anatomical elements and manual measurements observed in Mously et al., Pelekanos et al., and Borba et al. protocols are unclear and introduced measurements errors.

3.3. The Issue of Choosing the Micro-CT Scan Spatial Resolution. The unit element of a stack of images obtained by micro-CT scan is the voxel (volumetric pixel), the size of which depends on the X-ray magnification but is usually in the range of several microns to several tenth microns. The spatial resolution of the micro-CT scan is defined by the minimal dimensional variation that the micro-CT scan can acquire. This resolution is different from the image resolution given by voxel size. Voxel size can be lower than the spatial resolution. The spatial resolution is the combination of the detector resolution, the mechanical accuracies of the rotating, and sliding movements and the beam intensity [31]. The spatial resolution of the micro-CT scan must be chosen as well as a function of the range of crown adaptation parameters defined during prosthesis design about 50 to $100 \mu \mathrm{m}$.

3.4. The Problem of Standardizing Prosthetic Assembly Manufacturing. In the protocols of Borba et al. and Pelekanos et al. $[16,17]$, the prosthetic assembly was composed of a single prepared tooth model and crown copings obtained after CAD/CAM and sintering. However, different plaster copings of this prepared model were used to manufacture each crown coping. Therefore, the geometry of the assembly measured was not the same as the assembly manufactured by the CAD/CAM system to be evaluated. Plaster copings of the prepared tooth model introduce geometrical defects, so these defects were also evaluated on the crown adaptation. In the protocol of Mously et al. [18], they paired a prepared tooth coping and a crown coping obtained after CAD/CAM and sintering to compose the prosthetic assembly. Acrylic tooth copings of the tooth model were fabricated by CAD/CAM. Due to this duplication, geometrical defects were introduced by the first CAD/CAM system that was not subjected to evaluation. The range of these defects was unknown and assumed to be equivalent to those of the second CAD/CAM system to be evaluated. Each acrylic tooth was associated with its own CAD/CAM crown coping, so two consecutive $\mathrm{CAD} / \mathrm{CAM}$ systems and sintering were evaluated in this protocol.

The sintering step and the duplication of the tooth preparation model must be removed from the protocol to minimize the introduction of parasitic geometrical defects on the final adaptation measurements. As there is a gap between the prepared tooth coping and the prosthesis coping, several relative positions can be achieved between the two parts of the prosthetic assembly. The nonunicity of the prosthetic assembly is also a drawback when standardizing the protocol.

\section{X-Ray Micro-CT: 3D Metrological Chain Proposition-Pilot Study}

In this section, a new $3 \mathrm{D}$ metrological chain is proposed and validated on two simplified geometries of dental assembly which have the main characteristics of real dental assemblies. The need of standardized geometries is crucial in order to assess and compare CAD/CAM systems in the future.

Our method used a maximal covering sphere algorithm to assess a new indicator: Gap Size Distribution (GSD), which plays a key role in the quality of the assembly.

4.1. Reference Assembly: Standardized Geometries. Based on classical preparation recommendations, two simplified geometries are proposed (Figure 7) as those proposed by Borba et al. [16]. A pyramidal and a conical geometry are chosen. A crucial property of these geometries is that they are composed of regular shapes with PA defined mathematically: the axis of symmetry. Once defined, they are then designed using CAD software in this case Catia V5 (Dassault Systems).

The standardized prepared tooth is designed according to molar anatomical volume [26] and fixed prosthodontics clinical recommendations [27] as follows:

(i) The basis surface (modeling sound tissues of the tooth): parallelepiped with a square basis $(8 \times 8 \times$ $4 \mathrm{~mm}^{3}$ ) for the pyramidal part and cylinder basis (diameter of $8 \mathrm{~mm}$ and height of $4 \mathrm{~mm}$ ).

(ii) The margin curve (as defined in Figure 2): planar curves, respectively, a square of $8 \mathrm{~mm}$ length and a circle of $8 \mathrm{~mm}$.

(iii) The axial surface (as defined in Figure 2): respectively, a drafted pyramidal surface with a squared basis of 6 $\times 6 \mathrm{~mm}^{2}$, height of $6 \mathrm{~mm}$ and draft angle of $6^{\circ}$ and a drafted conical surface with a circular basis of $6 \mathrm{~mm}$ diameter, and height of $6 \mathrm{~mm}$ and draft angle of $6^{\circ}$. 

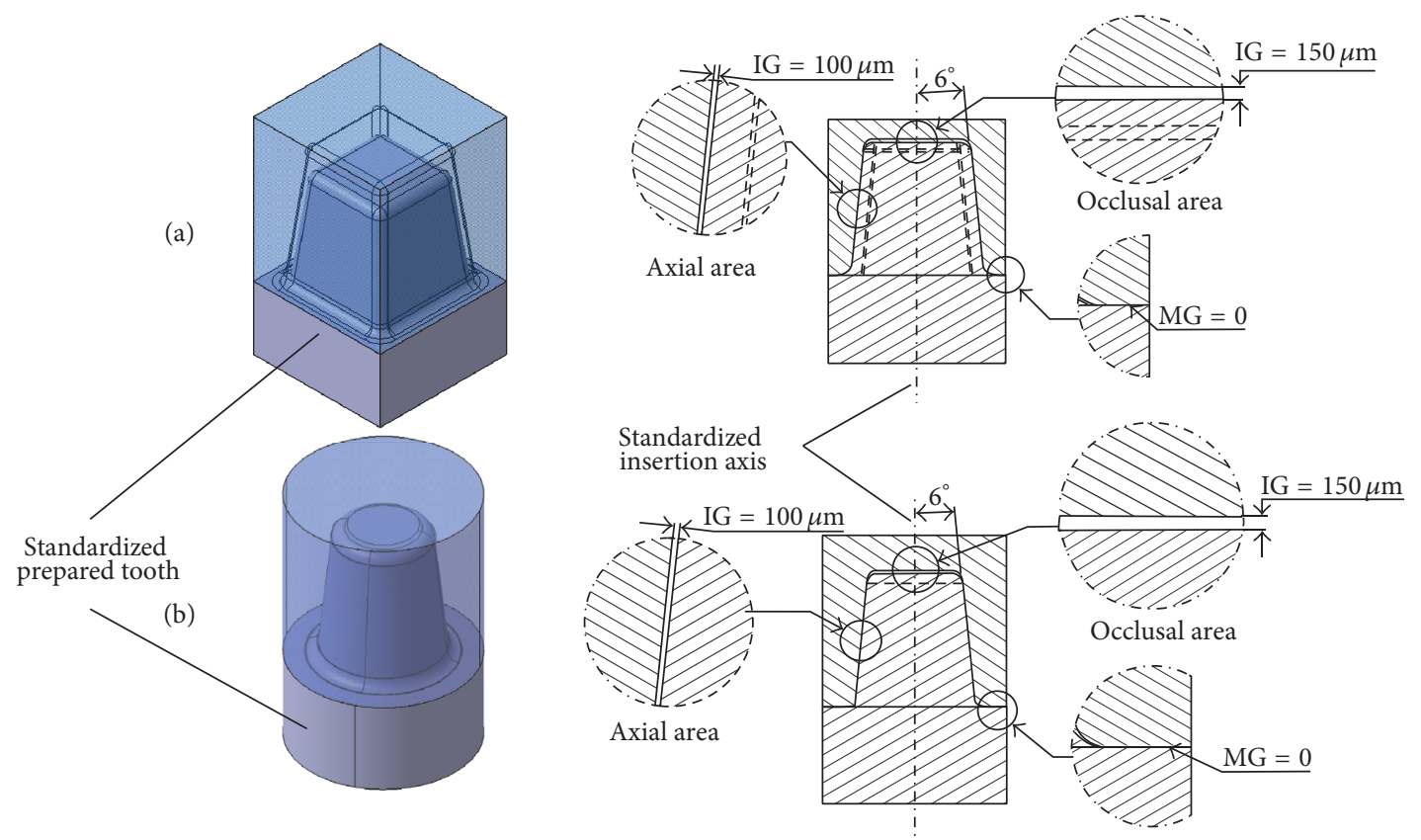

Figure 7: Standardized geometries: (a) pyramidal and (b) conical.

(iv) The marginal and occlusal surfaces are the planes, respectively, representing the basis and axial limiting surfaces.

(v) Marginal and occlusal surfaces are connected with rounded chamfers of $0.5 \mathrm{~mm}$ radius to the axial surface.

The standardized prostheses are designed according to the CAD/CAM design protocol. The axis of symmetry of the standardized prepared tooth (cone axis for the conical part and axis given by the intersection of the symmetry plane for the pyramidal part) is considered as PA.

The internal gap (IG in Figure 2) value is set to $100 \mu \mathrm{m}$ on the axial area and $150 \mu \mathrm{m}$ on the occlusal area according to the mean value considered clinically reliable $[6,32,33]$.

The marginal gap ( $\mathrm{MG}$ in Figure 2) value is set to zero, to avoid secondary caries due to microleakage in the marginal area, leading to the introduction of bacteria and gum irritation [34-36].

Likewise, no over- or underextended margins are manufactured. Over and underextended margins introduce dental plaque retention involving the accumulation of bacteria close to the marginal area and gum irritation. Thus, the underextended margin (UM) and overextended margin (OM) are set to zero (Figure 2). It results in the fact that the vertical marginal discrepancy (VMD), horizontal marginal discrepancy (HMD), and absolute marginal discrepancy (AMD) are null.

4.2. Reference Assembly: Materials and Fabrication. The two parts of the assembly (die and restoration) were made of a hybrid composite (Vita Enamic ${ }^{\circledR}$, Vita-Zahnfabrik, Germany: resin infiltrated ceramics). This biomaterial has several advantages with regard to other dental materials: good machinability, less brittle behavior than ceramics, and enhanced wear resistance compared to composites. Moreover, because of its good radioopacity, artifacts involved during X-ray acquisition are reduced.

The two standard assemblies were fabricated with a numerical-controlled milling machine prototype (Lyra, GACD).

4.3. X-Ray Micro-CT Acquisition. The CT images of the two types of assembly have been created with an X-ray microtomograph (X-View X50-CT, North Star Imaging) equipped with a cooled open microfocus a Dual Head XWT240XC190 tubes used in mode reflection less than from and a flat panel detector NSI Dexala $752\left(9.9^{\prime \prime} \times 11.4^{\prime \prime}, 75 \mathrm{~mm}\right.$ pixel pitch, $3073 \times 3888$ pixel matrix, dynamic range $14 \mathrm{bit})$. For the acquisition, reflection mode configuration of the $\mathrm{X}$-ray source was used (detail detectability less than $1 \mu \mathrm{m}$ ) with an accelerating voltage of $130 \mathrm{KV}$, a current of $50 \mu \mathrm{A}$. The detector was used in $2 \times 2$ binning mode and the exposure time was $200 \mathrm{~ms}$ per frame. A stack of roughly 1300 grayscale images was obtained from 1200 projections using a standard filtered back-projection reconstruction algorithm. The reconstruction of the CT image using a standard filtered back-projection reconstruction algorithm was performed with the NSI in-house software efX-CT. The magnification adopted allowed us to create CT image with a voxel size of approximately $8 \times 8 \times 8 \mu \mathrm{m}^{3}$ which is consistent for measuring a gap of $100 \mu \mathrm{m}$.

The image stack acquired was also processed using Avizo the $3 \mathrm{D}$ analysis software commercialized by FEI (Visualization Sciences Group) to visualize the assembly and the open source image-processing software ImageJ [37] to measure the 


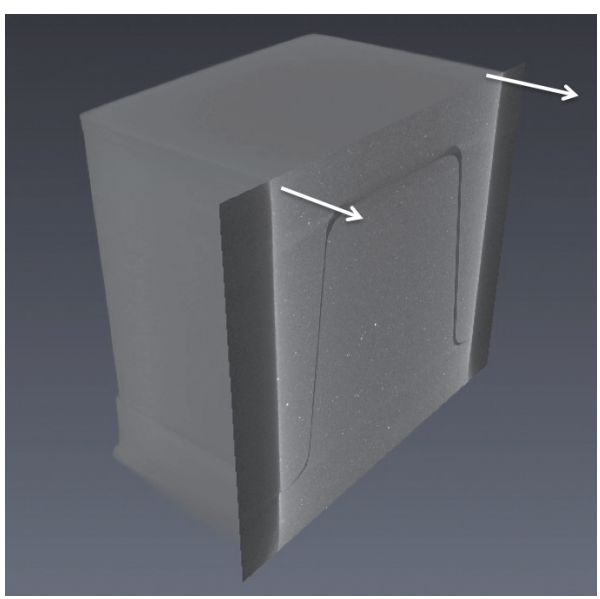

(a)

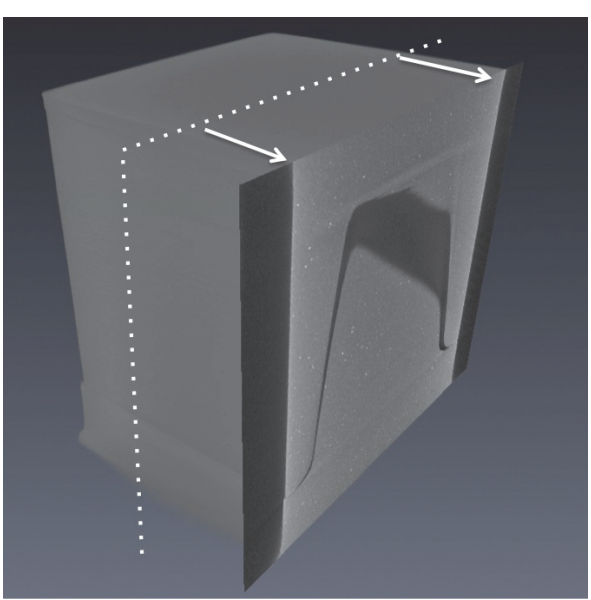

(b)

FIGURE 8: Direct 3D visualization of the pyramidal assembly. (a) A clipping plane has been added in the middle of the assembly. (b) A clipping plane has been added at a more marginal location.

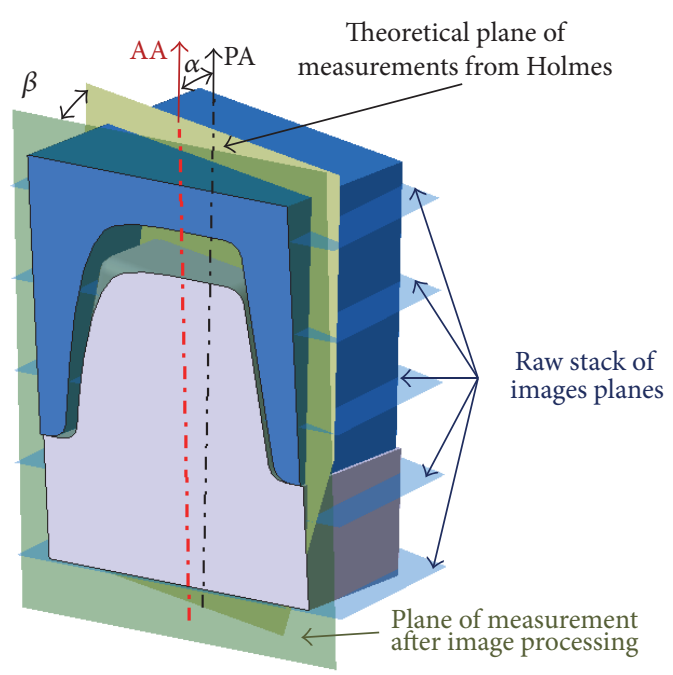

(a)

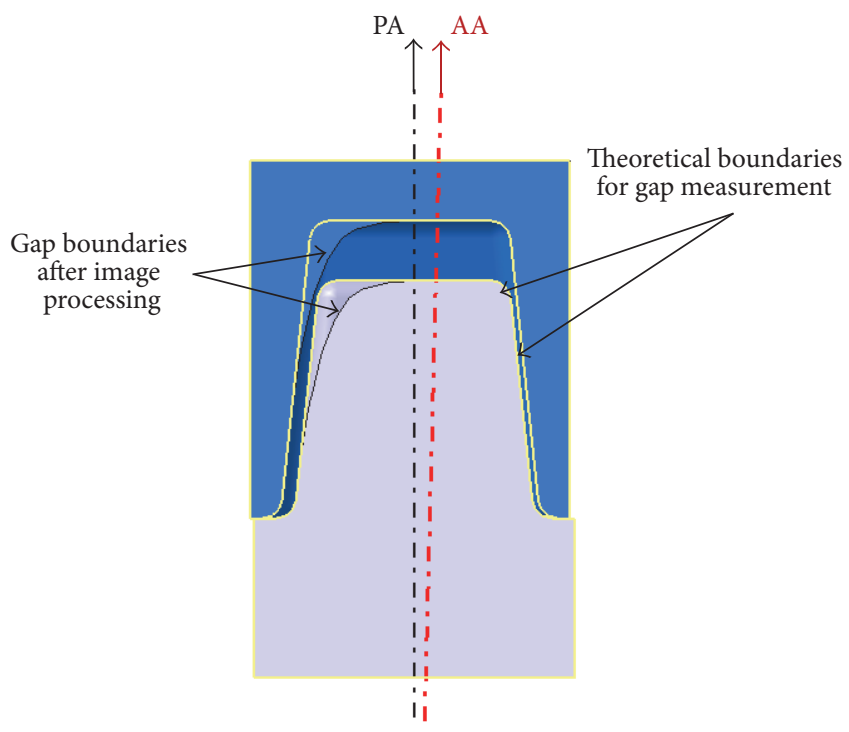

(b)

FIGURE 9: 3D simulation of errors occurring during CT scan acquisition and image postprocessing for pyramidal assembly (a) detailed errors; (b) overlay of theoretical and simulated image slices.

gap in 3D. A maximal covering sphere (MSC) algorithm was used $[38,39]$ to perform the $3 \mathrm{D}$ gap measurement.

4.4. 3D Visualization. An initial visual inspection of the assembly was conducted on the micro-CT images using Avizo's volume rendering tool (with threshold manual adjustment) and a vertical clipping plane at different locations was added in order to visualize the raw data (Figure 8 and film). It can be observed that the symmetry axis of the assembly (PA) was not perfectly coincident with the axis of acquisition (AA) in the tomograph, resulting in unexploitable vertical $2 \mathrm{D}$ images (Figure 8(b)). As discussed in Section 3, gap measurements cannot be processed directly from the raw stack of $2 \mathrm{D}$
micro-CT images. Thus, each surface (axial wall, internal surface), curve (prepared tooth and prosthesis margins), and measurement system of reference (PA and stack of reslicing planes) needed to perform the gap measurement were tilted from their theoretical position (Figure 9).

As for the protocols mentioned in Section 3, the insertion axis (PA, black axis in Figure 9), equivalent to the symmetry axis for our standard geometries, does not perfectly coincide with the tomograph acquisition axis (AA, red axis in Figure 9). The assembly positioning on the micro-CT scan object support introduces an angular misalignment ( $\alpha$ in Figure 9(a)): to avoid assembly parasitic movement during CT scanning the die was bonded on the support introducing 


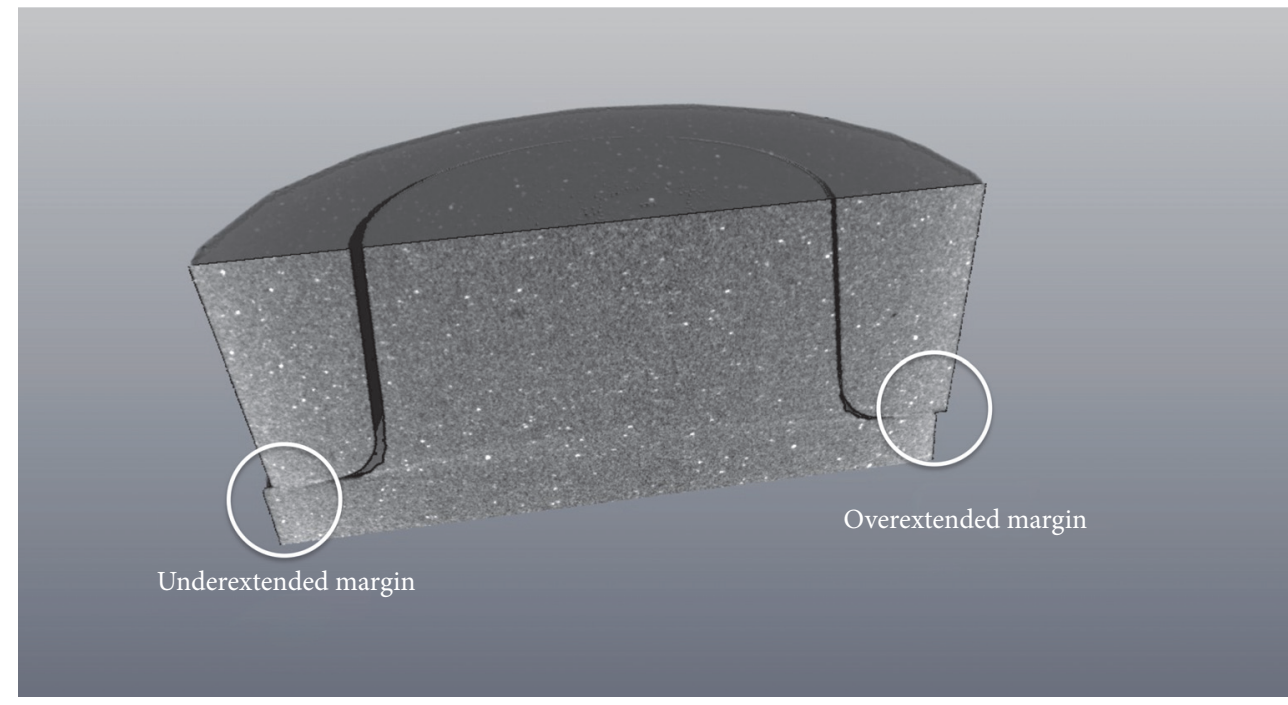

FIGURE 10: Direct 3D visualization of margin over- and underextension, due to misalignment of the prepared tooth and prosthesis axis.

positioning inaccuracy. As illustrated in Figure 9(a), the measurement plane (green plane) is obtained with an orthoslice of the raw stack of images (perpendicular to the blue planes directions AA); thus, the green plane is parallel from AA direction and tilted with $\alpha$ angle from the theoretical plane of measurements (beige vertical planes). Besides, during the orthoslice processing, the slicing directions must be defined introducing an angular misalignment $\beta$; thus, the green plane of measurements is also tilted with a $\beta$ angle from the theoretical plane of measurement (beige vertical planes). This double tilting results in inaccurate representation of the gap boundaries as illustrated in Figure 9(b). As explained previously, for the conical assembly, $\beta$ angle misalignment has no influence due to revolution axis of symmetry.

The margin over- and underextension can also be detected by direct $3 \mathrm{D}$ visualization, as depicted in Figure 10.

Under- and overextension result from a wrong positioning of the prosthesis when assembling. The prosthesis and the prepared tooth are in planar contact. Due to the gap between the two parts, as illustrated in Figure 11(a), one rotation around PA and two sliding movements, respectively, along $X_{0}$ and $Y_{0}$ directions are occurring. The sliding movements introduce a misalignment of the prosthesis (green axis in Figure 11) from PA used for the prosthetic assembly design (black axis in Figure 11). The combination of axis misalignment and prosthesis rotation generates over- and underextension of the prosthesis margin and nonuniform gap volume as illustrated in Figure 11(b). For the conical assembly, the rotation around PA has no influence due to revolution axis of symmetry.

The influence of the qualitative inspection and simulation of positioning defects on the gap measurement emphasizes the need to identify the assembly axis of symmetry (PA) and the direction of slicing plane before performing 2D gap measurements on the data acquired.
Besides, due to the gap, several positions of the prosthesis can be reached thus generating under- and overextension and nonuniform gap volume around the preparation. This gap volume distribution around the prepared tooth is difficult to analyze without a 3D characterization.

The conical assembly, due to its revolution axis of symmetry, allows avoiding problems of $2 \mathrm{D}$ measurement plane directions and relative rotation between the prosthesis and its die. Yet, the conical shape is less characteristic than the pyramidal shape regarding the prepared tooth shape obtained in clinical practice.

As standardizing the gap measurement protocol using the $2 \mathrm{D}$ measurements can be difficult, a new $3 \mathrm{D}$ method for measuring this gap is proposed.

4.5. New 3D Gap Measurement Method. To prevent errors arising from 2D measurements or limitations induced by images processing and especially reslicing, a maximal covering sphere algorithm is used in order to measure the gap in $3 \mathrm{D}$. The principle of this algorithm is to measure the dimension of a space at a point by finding the largest sphere containing the point (and remaining in the space considered) diameter which is called the maximal covering sphere (MCS). This algorithm is frequently used to measure the size of a solid/porous phase of porous media such as trabecular bone or scaffolds for tissue engineering [40]. The measure in $3 \mathrm{D}$ avoids the $2 \mathrm{D}$ errors that can be made (e.g., the second image in Figure 8 will lead to an overestimated gap size measurement). The MCS algorithm is used directly on the binarized stack of images acquired. So here, the main influencing parameter is the chosen threshold used to achieve binarization and the possible filters used to process the image stack. 


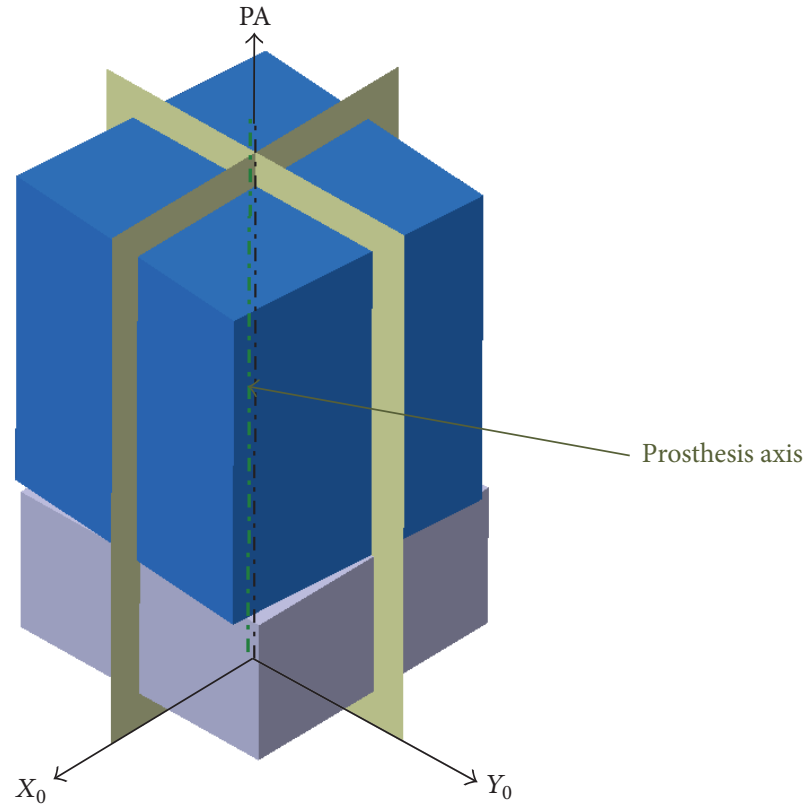

(a)

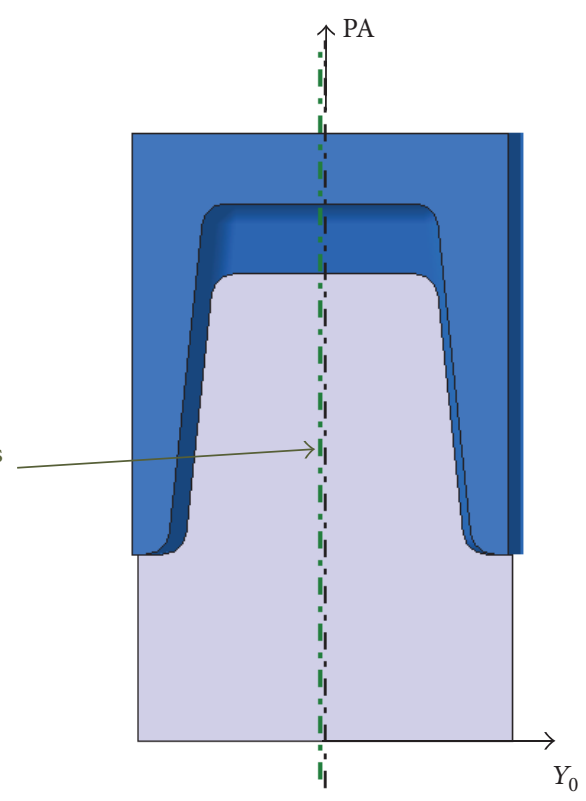

(b)

FIGURE 11: 3D simulation of errors occurring in prosthesis assembly.

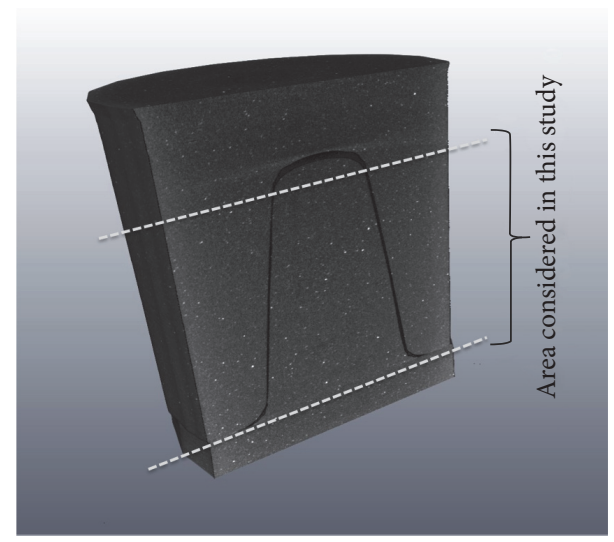

FIGURE 12: Area chosen to measure the gap in 3D illustrated on the conical assembly.

In this study, the proposed method is illustrated on the assembly and focuses on the gap axial and marginal areas (Figure 12).

Figure 13 represents the gap size map assessed by the MCS algorithm on assemblies. The gap measurement is achieved on the whole volume of the gap and not only on a few points on a few slices.

Figure 14 shows the histogram of the Gap Size Distribution (GSD) in these assemblies (the indicated frequency corresponding to the probability to find this gap size). The conical assembly (gray bars) exhibits a smooth Gap Size Distribution from 0 to $200 \mu \mathrm{m}$, whereas a bimodal Gap Size Distribution is detected in the pyramidal assembly. The pyramidal assembly presents two main gap sizes around $50 \mu \mathrm{m}$ and around $140 \mu \mathrm{m}$.

The figures clearly indicate a translation of the prosthetic part assemblies. This translation explains the overand underextension observed in Figure 10. This translation explains the over- and underextension observed in Figure 10. It results in a heterogeneous gap whose dimension varies by up to $200 \mu \mathrm{m}$ in both types of assembly. As the assembly was designed to have a homogeneous gap of $100 \mu \mathrm{m}$, it is logical to have a gap up to $200 \mu \mathrm{m}$ due to the translation of the prosthesis (leading to a minimal gap at one point and to a "double" gap at the opposite point).

The conical assembly gap size is more smoothly distributed and we predict that such smooth distribution will be more favorable for the durability of the prosthesis through time (of course, further studies are needed to demonstrate this).

This therefore leads to the first interesting conclusion that can be drawn from this study: different tooth preparations will lead to different GSDs, thereby certainly influencing the future of prosthesis life. It should be interesting to measure the GSD in the case where the gap is filled (by an adhesive or a cement), then to observe whether it is more homogeneous, and finally to assess the impact of the GSD on the mechanical behavior of the assembly.

We may also remark that

(i) the 3D map of the gap size can also allow the detection of a rotation of the prosthesis on the die,

(ii) the MCS algorithm can also be used to measure the thickness of the prosthesis. Another indicator that can be called PTD (Prosthesis Thickness Distribution) can be defined. 


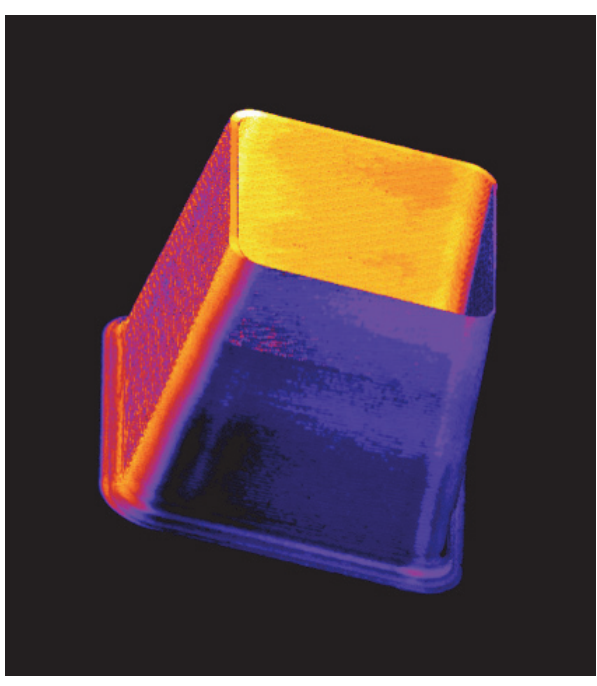

(a)

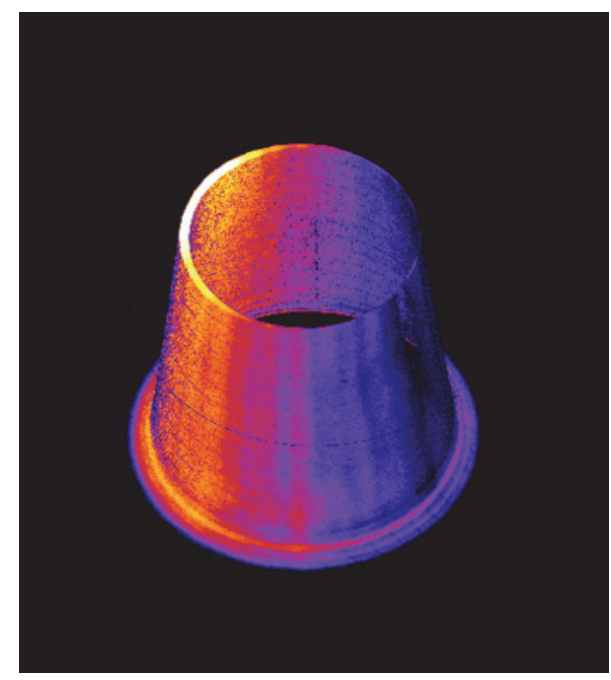

(b)

FIgURE 13: 3D visualization of gap dimension (obtained using the MCS algorithm). (a) Pyramidal shape. (b) Conical shape. White color corresponds to the maximal gap size of each case.

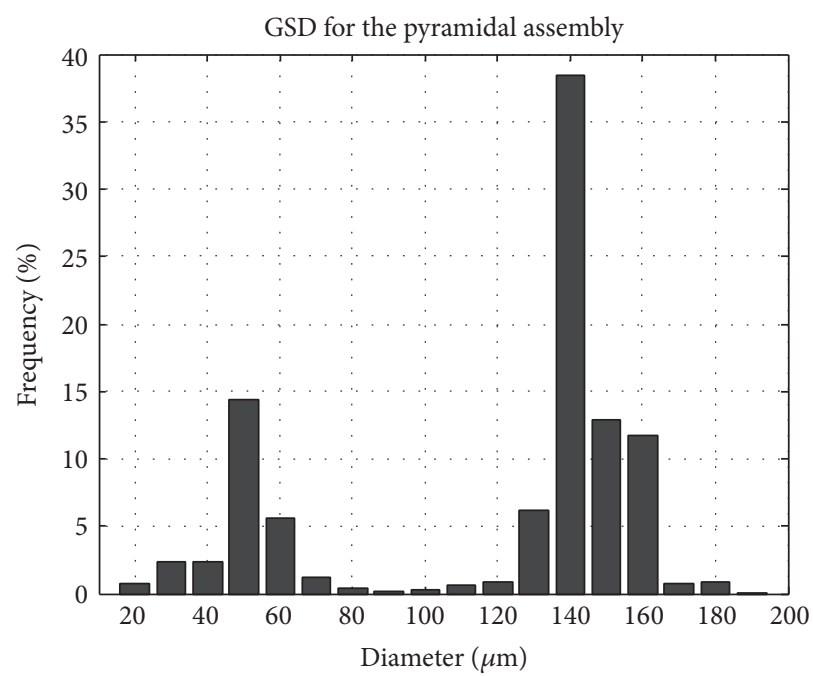

(a)

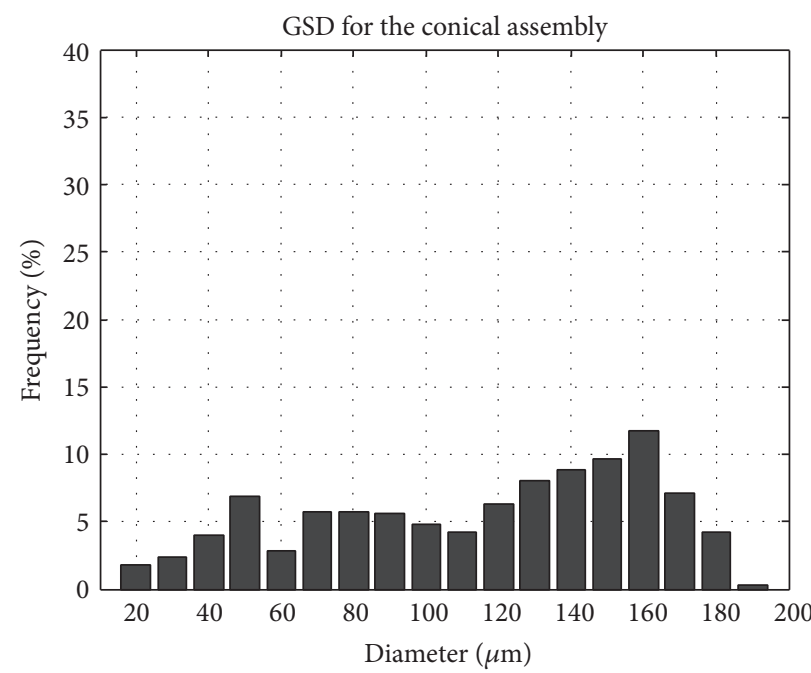

(b)

FIgURE 14: Gap Size Distribution (GSD) for the pyramidal assembly (a) and for the conical assembly (b).

\section{Conclusion}

In this paper, a critical review of the protocols using $\mathrm{X}$-ray micro-CT to evaluate a prosthetic assembly adaptation was proposed and used to define a new 3D protocol.

Reference geometries mimicking a prosthetic assembly had to be defined to compare different production systems. Two parameterized geometries were defined and used to validate our gap 3D measurement protocol (a conical and a pyramidal assembly). A 2D gap measurement protocol was also mentioned, but we argued that this type of measurement is extremely sensitive to the choice and definition of the measurement plane. Resulting plane choice errors were simulated and quantified in order to highlight the difficulty of using such a protocol, especially on a real dental prosthetic assembly.

By definition, the $3 \mathrm{D}$ measurement protocol helps to overcome this problem: plane definition is no longer needed and so the 3D measurement protocol can be used on all types of prosthetic assembly. It also allows the estimation of a new indicator, Gap Size Distribution (GSD), which is a representation of the gap size in the whole area considered. The GSD in a conical and a pyramidal assembly was represented and presented an interesting difference: the GSD in the conical assembly provided smooth distribution whereas in the pyramidal assembly distribution preparation was clearly 
bimodal. Therefore, the natural next step of this study is to investigate the influence of GSD on the future life of the prosthesis, especially on its mechanical behavior.

\section{Competing Interests}

The authors declare that there is no conflict of interests regarding the publication of this paper.

\section{Acknowledgments}

This work benefited from the financial support of the LabeX LaSIPS (ANR-10-LABX-0040-LaSIPS), managed by the French National Research Agency under the "Investissements d'Avenir" program (no. ANR-11-IDEX-0003-02). This work also received financial support from the French "Agence Nationale de la Recherche," through the "Investissements d'Avenir" program (ANR-10-EQPX-37 MATMECA Grant). The authors would like to thank Mr. N. Lenoir (Laboratoire Navier, UMR 8205) and Mr. B. Smaniotto (LMT Cachan, UMR8535) for their technical assistance in performing the micro-CT acquisitions.

\section{References}

[1] D. J. Fasbinder, "Computerized technology for restorative dentistry," American Journal of Dentistry, vol. 26, no. 3, pp. 115-120, 2013.

[2] R. van Noort, “The future of dental devices is digital," Dental Materials, vol. 28, no. 1, pp. 3-12, 2012.

[3] T. Miyazaki, Y. Hotta, J. Kunii, S. Kuriyama, and Y. Tamaki, "A review of dental CAD/CAM: current status and future perspectives from 20 years of experience," Dental Materials Journal, vol. 28, no. 1, pp. 44-56, 2009.

[4] F. Beuer, J. Schweiger, and D. Edelhoff, "Digital dentistry: an overview of recent developments for CAD/CAM generated restorations," British Dental Journal, vol. 204, no. 9, pp. 505-511, 2008.

[5] A. Merdji, B. Bachir Bouiadjra, B. Ould Chikh et al., "Stress distribution in dental prosthesis under an occlusal combined dynamic loading," Materials and Design, vol. 36, pp. 705-713, 2012.

[6] P. Boitelle, B. Mawussi, L. Tapie, and O. Fromentin, "A systematic review of CAD/CAM fit restoration evaluations," Journal of Oral Rehabilitation, vol. 41, no. 11, pp. 853-874, 2014.

[7] K. Kuhn, S. Ostertag, M. Ostertag, M. H. Walter, R. G. Luthardt, and H. Rudolph, "Comparison of an analog and digital quantitative and qualitative analysis for the fit of dental copings," Computers in Biology and Medicine, vol. 57, pp. 32-41, 2015.

[8] S. Karlsson, "The fit of procera titanium crowns: an in vitro and clinical study," Acta Odontologica Scandinavica, vol. 51, no. 3, pp. 129-134, 1993.

[9] M. K. Molin and S. L. Karlsson, "A randomized 5-year clinical evaluation of 3 ceramic inlay systems," International Journal of Prosthodontics, vol. 13, no. 3, pp. 194-200, 2000.

[10] K. Tan, B. E. Pjetursson, N. P. Lang, and E. S. Y. Chan, "A systematic review of the survival and complication rates of fixed partial dentures (FPDs) after an observation period of at least 5 years," Clinical Oral Implants Research, vol. 15, no. 6, pp. 654666, 2004.

[11] J.-G. Wittneben, R. F. Wright, H.-P. Weber, and G. O. Gallucci, "A systematic review of the clinical performance of CAD/CAM single-tooth restorations," The International Journal of Prosthodontics, vol. 22, no. 5, pp. 466-471, 2009.

[12] L. M. Galantucci, R. Ferrandes, and G. Percoco, "Digital photogrammetry for facial recognition," Journal of Computing and Information Science in Engineering, vol. 6, no. 4, pp. 390-396, 2006.

[13] L. M. Galantucci, G. Percoco, F. Lavecchia, and E. Di Gioia, "Noninvasive computerized scanning method for the correlation between the facial soft and hard tissues for an integrated three-dimensional anthropometry and cephalometry," Journal of Craniofacial Surgery, vol. 24, no. 3, pp. 797-804, 2013.

[14] E. Vennat, W. Wenlong, A. Gourrier, R. Genthial, and J. P. Attal, "3D imaging of the dentinal porous structure using confocal laser scanning microscopy," in Proceedings of the 4th International Conference on Computational and Mathematical Biomedical Engineering, Cachan, France, June 2015.

[15] L. M. Galantucci, G. Percoco, F. Lavecchia, and E. Di Gioia, "Noninvasive computerized scanning method for the correlation between the facial soft and hard tissues for an integrated three-dimensional anthropometry and cephalometry," Journal of Craniofacial Surgery, vol. 24, no. 3, pp. 797-804, 2013.

[16] M. Borba, P. F. Cesar, J. A. Griggs, and Á. Della Bona, "Adaptation of all-ceramic fixed partial dentures," Dental Materials, vol. 27, no. 11, pp. 1119-1126, 2011.

[17] S. Pelekanos, M. Koumanou, S.-O. Koutayas, S. Zinelis, and G. Eliades, "Micro-CT evaluation of the marginal fit of different In-Ceram alumina copings," The European Journal of Esthetic Dentistry, vol. 4, no. 3, pp. 278-292, 2009.

[18] H. A. Mously, M. Finkelman, R. Zandparsa, and H. Hirayama, "Marginal and internal adaptation of ceramic crown restorations fabricated with CAD/CAM technology and the heat-press technique," Journal of Prosthetic Dentistry, vol. 112, no. 2, pp. 249-256, 2014.

[19] O. Schaefer, D. C. Watts, B. W. Sigusch, H. Kuepper, and A. Guentsch, "Marginal and internal fit of pressed lithium disilicate partial crowns in vitro: a three-dimensional analysis of accuracy and reproducibility," Dental Materials, vol. 28, no. 3, pp. 320-326, 2012.

[20] O. Moldovan, R. G. Luthardt, N. Corcodel, and H. Rudolph, "Three-dimensional fit of CAD/CAM-made zirconia copings," Dental Materials, vol. 27, no. 12, pp. 1273-1278, 2011.

[21] J. Ng, D. Ruse, and C. Wyatt, "A comparison of the marginal fit of crowns fabricated with digital and conventional methods," Journal of Prosthetic Dentistry, vol. 112, no. 3, pp. 555-560, 2014.

[22] J. P. Kruth, M. Bartscher, S. Carmignato, R. Schmitt, L. De Chiffre, and A. Weckenmann, "Computed tomography for dimensional metrology," CIRP Annals-Manufacturing Technology, vol. 60, no. 2, pp. 821-842, 2011.

[23] J. R. Holmes, S. C. Bayne, G. A. Holland, and W. D. Sulik, "Considerations in measurement of marginal fit," The Journal of Prosthetic Dentistry, vol. 62, no. 4, pp. 405-408, 1989.

[24] "The glossary of prosthodontic terms," Journal of Prosthetic Dentistry, vol. 94, no. 1, pp. 10-92, 2005.

[25] W. A. Kalender, "X-ray computed tomography," Physics in Medicine and Biology, vol. 51, no. 13, pp. R29-R43, 2006.

[26] J. B. Woelfel and R. C. Scheid, Dental Anatomy: Its Relevance to Dentistry, Williams \& Wilkins, 2002. 
[27] H. Wiskott, Fixed Prosthodontics: Principles and Clinics, Quintessence, 2011.

[28] S. Siddique, M. Imran, M. Rauer et al., "Computed tomography for characterization of fatigue performance of selective laser melted parts," Materials and Design, vol. 83, pp. 661-669, 2015.

[29] M. Ito, "Assessment of bone quality using micro-computed tomography (micro-CT) and synchrotron micro-CT," Journal of Bone and Mineral Metabolism, vol. 23, no. 1, pp. 115-121, 2005.

[30] R. Giordano, "Materials for chairside CAD/CAM-produced restorations," Journal of the American Dental Association, vol. 137, no. 9, pp. 14-31, 2006.

[31] C. T. Badea, A. W. Wetzel, N. Mistry, S. Pomerantz, D. Nave, and G. A. Johnson, "Left ventricle volume measurements in cardiac micro-CT: the impact of radiation dose and contrast agent," Computerized Medical Imaging and Graphics, vol. 32, no. 3, pp. 239-250, 2008.

[32] I. Sailer, A. Fehér, F. Filser et al., "Prospective clinical study of zirconia posterior fixed partial dentures: 3-year follow-up," Quintessence International, vol. 37, no. 9, pp. 685-693, 2006.

[33] I. Sailer, A. Fehér, F. Filser, L. J. Gauckler, H. Lüthy, and C. H. F. Hämmerle, "Five-year clinical results of zirconia frameworks for posterior fixed partial dentures," International Journal of Prosthodontics, vol. 20, no. 4, pp. 383-388, 2007.

[34] W. Romão Jr., W. G. Miranda Jr., P. F. Cesar, and R. R. Braga, "Correlation between microleakage and cement thickness in three Class II inlay ceramic systems," Operative Dentistry, vol. 29, no. 2, pp. 212-218, 2004.

[35] P. H. O. Rossetti, A. L. do Valle, R. M. de Carvalho, M. F. de Goes, and L. F. Pegoraro, "Correlation between margin fit and microleakage in complete crowns cemented with three luting agents," Journal of Applied Oral Science, vol. 16, no. 1, pp. 64-69, 2008.

[36] C. P. Trajtenberg, S. J. Caram, and S. Kiat-Amnuay, "Microleakage of all-ceramic crowns using self-etching resin luting agents," Operative Dentistry, vol. 33, no. 4, pp. 392-399, 2008.

[37] W. Rasband, ImageJ, Bethesda, Md, USA.

[38] T. Hildebrand and P. Rüegsegger, "A new method for the modelindependent assessment of thickness in three-dimensional images," Journal of Microscopy, vol. 185, no. 1, pp. 67-75, 1997.

[39] R. Dougherty and K. Kunzelmann, "Computing local thickness of 3D structures with imageJ," Microscopy and Microanalysis, vol. 13, no. S02, pp. 1678-1679, 2007.

[40] M. A. Knackstedt, C. H. Arns, T. J. Senden, and K. Gross, "Structure and properties of clinical coralline implants measured via 3 D imaging and analysis," Biomaterials, vol. 27, no. 13, pp. 27762786, 2006. 

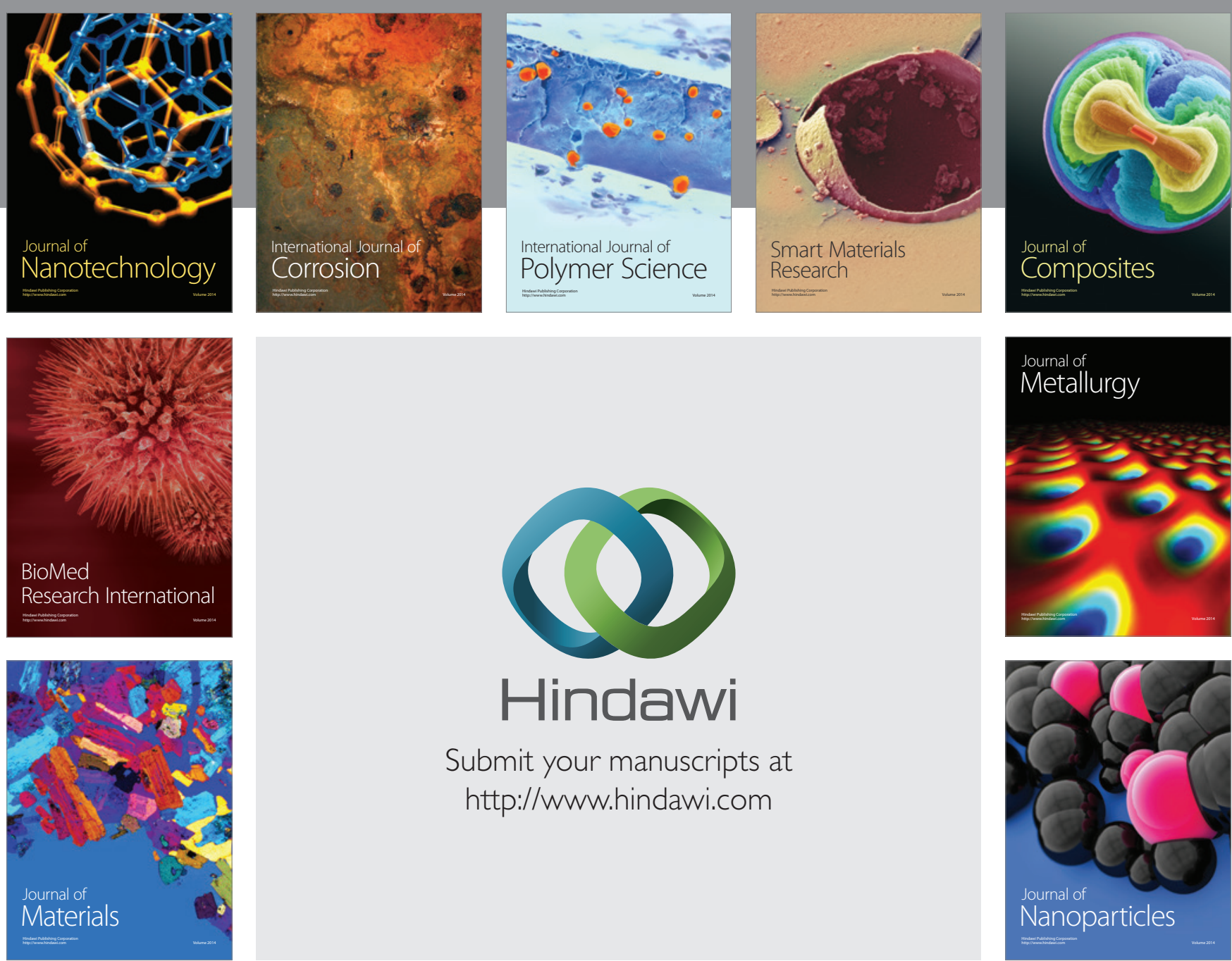

\section{Hindawi}

Submit your manuscripts at

http://www.hindawi.com

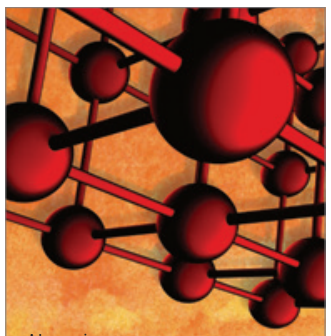

Materials Science and Engineering
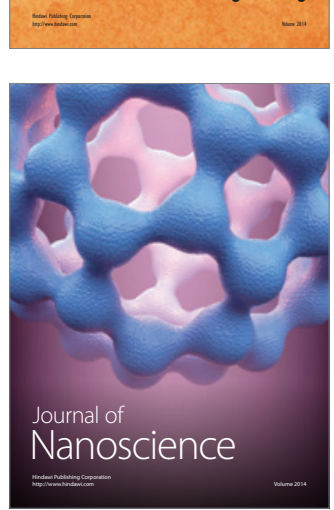
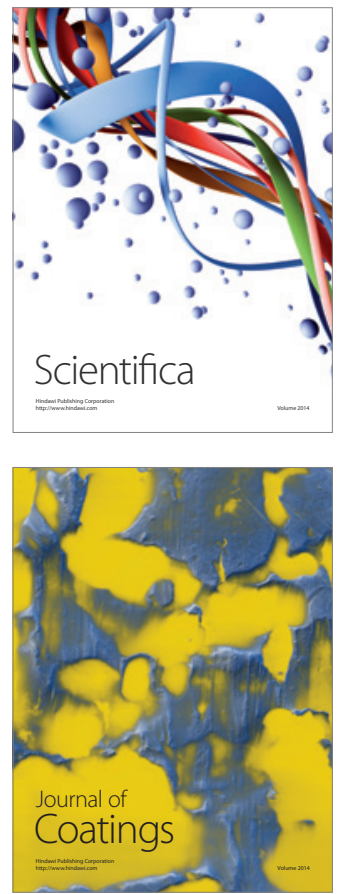
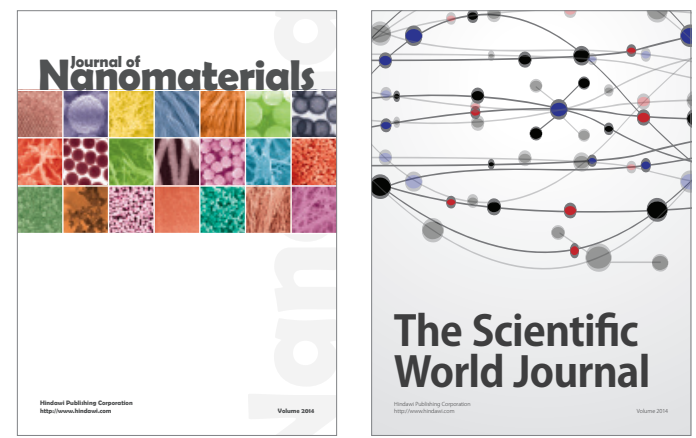

The Scientific World Journal
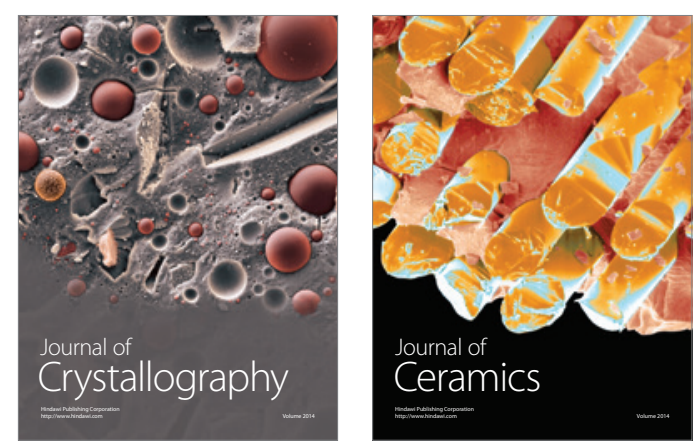
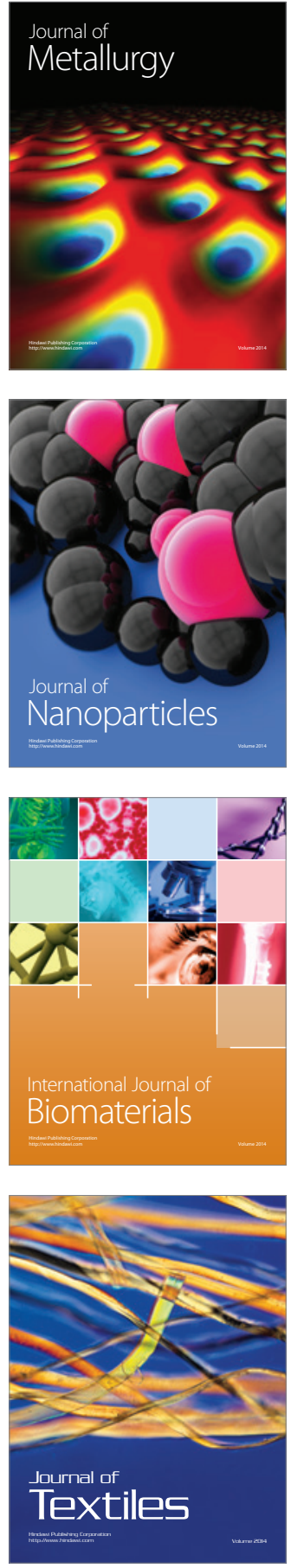